\title{
Research and development toward heavy ion driven inertial fusion energy
}

\author{
Peter A. Seidl, ${ }^{1}$ John J. Barnard, ${ }^{2}$ Andris Faltens, ${ }^{1}$ and Alex Friedman ${ }^{2}$ \\ ${ }^{1}$ Lawrence Berkeley National Laboratory, 1 Cyclotron Road, Berkeley, California 94720, USA \\ ${ }^{2}$ Lawrence Livermore National Laboratory, Mail Stop L-637, P.O. Box 808, Livermore, California 94551-0808, USA
}

(Received 13 June 2012; published 1 February 2013)

\begin{abstract}
We describe near-term heavy ion fusion (HIF) research objectives associated with developing an inertial fusion energy demonstration power plant. The goal of this near-term research is to lay the essential groundwork for an intermediate research experiment (IRE), designed to demonstrate all the key driver beam manipulations at a meaningful scale, and to enable HIF relevant target physics experiments. This is a very large step in size and complexity compared to HIF experiments to date, and if successful, it would justify proceeding to a demonstration fusion power plant. With an emphasis on accelerator research, this paper is focused on the most important near-term research objectives to justify and to reduce the risks associated with the IRE. The chosen time scale for this research is 5-10 years, to answer key questions associated with the HIF accelerator drivers, in turn enabling a key decision on whether to pursue a much more ambitious and focused inertial fusion energy research and development program. This is consistent with the National Academies of Sciences Review of Inertial Fusion Energy Systems Interim Report, which concludes that "it would be premature at the present time to choose a particular driver approach..." and encouraged the continued development of community consensus on critical issues, and to develop "options for a community-based roadmap for the development of inertial fusion as a practical energy source."
\end{abstract}

DOI: 10.1103/PhysRevSTAB.16.024701

\section{MOTIVATION}

There is renewed interest in the development of energy solutions that can provide carbon-free, base-load electricity. The National Ignition Facility (NIF) campaign of laserdriven fusion ignition experiments is under way [1]. The prospect of demonstrating laboratory-scale inertial fusion in the near term is stimulating interest in a variety of approaches to inertial fusion energy (IFE), such as laserdriven IFE and including heavy ion driven inertial fusion (HIF) energy. The various approaches involve heating the surface of a shell containing the deuterium-tritium (DT) fuel to drive an implosion which compresses and heats the fuel. This usually requires a driver energy $\geq 1$ MJ. Heavy ions of mass $\sim 100 \mathrm{amu}$ and ion kinetic energy $\geq 1 \mathrm{GeV}$ have a stopping range suitable to drive IFE targets. To satisfy a target design's particular ion range (and target performance) there is, a priori, a richness of possible combinations of ion mass and ion kinetic energy.

The three principal approaches to a heavy ion fusion driver are synchrotrons, radio-frequency linear accelerators (with storage rings), and induction linear accelerators. In general, accelerators operate reliably and can operate with high efficiency. The rf accelerators with storage rings are appealing because of their extensive use in high-energy

Published by the American Physical Society under the terms of the Creative Commons Attribution 3.0 License. Further distribution of this work must maintain attribution to the author(s) and the published article's title, journal citation, and DOI.
PACS numbers: 52.58.Hm, 89.30.Jj, 29.20.Ej, 41.75.-i

and nuclear physics. Induction accelerators are appealing because of their higher efficiency and their demonstrated acceleration of high beam current (10 kA in some applications). The U.S. effort has focused on induction accelerators because of this and because there is no need to accumulate charge in storage rings; their nonresonant character allows pulse compression during acceleration. Baseline driver design in the U.S. consists of about 100 parallel ion beams injected into an induction accelerator, with the bundle of beams passing through common induction accelerator cores. The beams are accelerated to a final kinetic energy of $1 \mathrm{GeV}$ per ion, or higher. Because of the high charge per bunch, transport is initially the limiting consideration, resulting from the need to provide sufficient focusing force to contain the space-charge force; a process that is facilitated by using multiple beams. The limit depends on the ion velocity, the peak focusing magnetic field, and the geometry of the focusing magnets. The approach is to accelerate these long parallel bunches near the transport limit and gradually decrease their length within the accelerator-as allowed by beam dynamicsby small voltage ramps. The transportable line charge density and the maximum current that can be transported in the space-charge-dominated regime increases with velocity because of the increasing strength of the $\boldsymbol{v} \times \boldsymbol{B}$ force.

A conceptual layout of a likely configuration, motivating much of the content of this paper, is shown in Fig. 1. Operating at $5-15 \mathrm{~Hz}$, about 100 parallel ion beams are injected into a linear induction accelerator. Other induction 


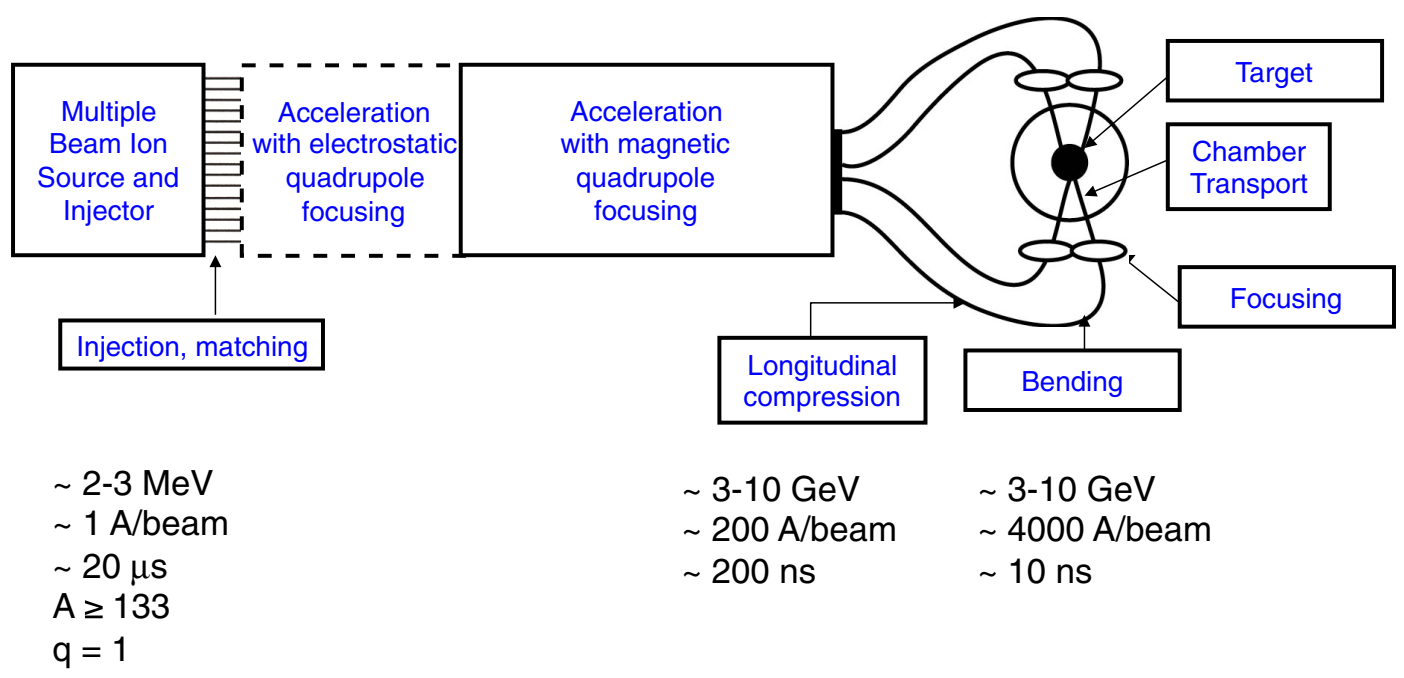

FIG. 1. Schematic of an induction accelerator driver for heavy ion fusion.

accelerator architectures have been studied, for example, separate accelerators for each beam, and recirculating induction accelerators. Initially motivated by their potential to lower cost, those design studies identified additional beam physics and technical issues, as described in Ref. [2].

Singly charged $(q=1)$ ions are often chosen because higher charge state ions create proportionally more space charge which would be much more difficult to produce and match to the alternating gradient lattice. Other favorable aspects of $q=1$ ions are the ability to create low-emittance beams of sufficiently high current with essentially no admixture of $q>1$ ions, and the lower longitudinal confinement fields required for bunch containment. Of course, a disadvantage is the proportionally lower acceleration rate.

The accelerator may use electrostatic focusing quadrupoles at the front end, followed by a transition to superconducting magnetic quadrupoles for most $(>90 \%)$ of the accelerator.

A velocity ramp is applied to the beam near the end of the accelerator. The beam $(\beta=0.2-0.3)$ is not highly relativistic, thus the bunch length is compressed by an order of magnitude or more and shaped as needed to meet the $\sim 10$ ns bunch duration required by most targets. A part of the drift compression section includes dipoles for each beam line to aim each beam at the target according to the required illumination geometry.

Equations governing the extraction of ions from sources, beam transverse dynamics, the transport to the reactor chamber, and the final focusing of the beams onto the target are described in various references. See, for example, [3-5] and Chapter 10 of [2]. Driver concepts and their design equations are summarized in Hovingh et al. [5] and applied to sensitivity studies of the cost of the fusion driver to assumed technical limits and beam properties.
At the wall of the reactor chamber, each beam line must penetrate the chamber wall while leaving sufficient solid angle for a viable tritium breeding blanket and heat extraction. This blanket design has been envisioned [see, e.g., [6]] as a flowing, thick, neutron-absorbing layer of lithium or a molten salt containing lithium, which protects both the structural wall and the focusing magnet coils from radiation damage. There are holes in the liquid matrix (created by an array of many liquid jets) through which the beams may pass. The magnet coils of the final optics are not on a line of sight to the target, and thus avoid the direct gamma rays, ions, neutrons, and target debris from the target explosion. Liquid-wall protection promises to be a highly attractive attribute of ion-beam driven IFE, provided the beam geometry is suitably arranged.

Not shown in Fig. 1 are the essential tritium extraction, target factory, heat recovery, and electricity generation systems. Many of the requirements and research for these systems is synergistic with laser IFE and some important differences are described later. Laser and HIF chamber and final focusing systems are designed to operate at a similar repetition rate, with each fuel pellet generating similar thermonuclear yield. The two approaches must be designed to protect the structural wall of the chamber, the final focusing elements, and the beam lines from backstreaming radiation and debris from the target.

Fundamental aspects of the fusion target designs have a great influence on the final beam parameters and target illumination geometry and, as a result, on the accelerator design. For example, the required beam energy per pulse varies among target designs by a factor of several, which will influence the number of parallel beams and other aspects of the accelerator design. Also, the beam pulse duration depends on the ignition mode, from $<0.5 \mathrm{~ns}$ to $\sim 10 \mathrm{~ns}$ depending on the target design. Most targets generally require a low power prepulse, with 20-100 ns 
duration to efficiently compress the fusion fuel prior to the main pulse.

This paper outlines research to answer key scientific and technical questions. Such a research program is a prerequisite for a heavy-ion accelerator facility capable of doing HIF target physics and demonstrating at driver scale the essential accelerator components of an HIF power plant, including beam control and focused beam intensity. Most of the accelerator research centers on the concept of an induction linear accelerator, producing beams with a final kinetic energy of $1 \mathrm{GeV}$ or higher. Given the combined need for accelerator efficiency and phase-space constraints set by most HIF target designs, and driver cost, this appears to be the best-matched accelerator architecture, with many of the system requirements already demonstrated for other applications.

We begin with a background description of the highlevel questions that frame the research agenda. This is followed by descriptions of the proposed research in several areas (with an emphasis on accelerator $R \& D$, since the accelerator is the long-lead-time item for heavy ion IFE). These topics include multiple-beam ion sources and injectors; studies of the injection and transport of a single driver-scale beam at $\sim 5-15 \mathrm{~Hz}$; studies of intense-beam transport in a system long enough that the ions execute several transverse plasma (beam) oscillations; studies of drift compression, bending, and final focus; studies of target physics; research on enabling technologies; and research opportunities that build on and contribute to related fields. We conclude with a brief summary.

\section{BACKGROUND}

The U.S. National Academies of Sciences and Engineering (NAS) are sponsoring a review of the prospects for inertial confinement fusion energy systems. This will include various driver systems, including heavy-ion accelerators. Presentations commenced early in 2011, and included detailed expositions of laser-driven IFE, pulsed power IFE, and heavy ion driven IFE $[7,8]$. The charges to the NAS review committee include the identification of R\&D objectives associated with developing an IFE demonstration plant, and to advise the DOE on its development of an R\&D roadmap aimed at creating a conceptual design for an inertial fusion energy demonstration plant. This IFE demonstration plant is sometimes referred to as a DEMO or fusion test facility, and in the HIF presentations to the NAS, was called an HIF Test Facility. (The term "fusion test facility" has sometimes been applied to a precursor to a DEMO. However, in IFE it may be upgraded in some cases to DEMO capability.) This demonstration plant should produce fusion power, breed tritium, and demonstrate all key scientific and engineering points.

Since the late 1990s the development path for HIF has included a prerequisite for the demonstration power plant: an intermediate facility, the objective of which is to demonstrate all the key driver beam manipulations at a meaningful scale, and to enable HIF relevant target physics experiments. If successful, this would lead to the design and construction of a DEMO plant. To that end, the goals for an integrated research experiment (IRE) for HIF are described in [4]. A laser based IRE was also an element of the common roadmap for heavy ion driven and laser-driven IFE [9]. Recently, objectives for a 10-kJ scale HIF IRE facility, called the heavy ion driven implosion experiment (HIDIX) have been described [10]. Both the earlier HIF IRE and the more recent HIDIX are in the same location in the development path for HIF-a significant milestone that precedes, and if successful, either would justify a fusion power DEMO. The proposed accelerator architectures are multiple-beam induction linear accelerators with predominantly magnetic quadrupole focusing. The main differences are that the design objective of the HIDIX seeks to achieve implosions with driver-relevant illumination geometry, including IFE chamber and target injection experiments, while using significantly lower beam pulse energy $(10 \mathrm{~kJ} v s \geq 30 \mathrm{~kJ})$. HIDIX has a top kinetic energy of at least $1.3 \mathrm{GeV}$, a maximum acceleration rate of $2 \mathrm{MV} / \mathrm{m}$, and assumes the development of a strong, nonlinear plasma based focusing element preceding the target to reduce the spot size from $\approx 2 \mathrm{~mm}$ to $0.1-0.5 \mathrm{~mm}$. In contrast, the IRE designs in $[3,4]$ are more conservative, with a top kinetic energy from $200-800 \mathrm{MeV}$, a maximum acceleration rate of 1-2 MV/m, a final spot size of $\leq 5 \mathrm{~mm}$, and $30-250 \mathrm{~kJ}$ beam pulses. IFE relevant target physics in a planar geometry are feasible for all those designs, and implosions for some of them.

We note that these various IRE (and HIDIX) design parameters imply a variety of scientific and technical capabilities and uncertainties. Furthermore, the cost uncertainties for these designs are large. But the overriding, and unifying goal, together with parallel progress on IFE chamber and target $R \& D$, is to provide the scientific and technological basis to proceed to the DEMO. It is highly desirable that the beam parameters and associated driver technology for this intermediate facility resemble those required for the DEMO in the most important aspects. The DEMO will in turn integrate all the major systems needed for an IFE power plant (driver, target production and injection, fusion chamber and heat removal). Thus, there are three general areas where the IRE must provide solid justification for the DEMO: (1) accelerator physics; (2) chamber transport and final focus; and (3) ion-beam interaction with targets.

Several of the key scientific questions related to HIF drivers have been answered: Experiments and simulations showed that stable beam transport over long distances in the accelerator can be achieved with low-emittance growth in the presence of high space charge. Experiments addressing most beam manipulations and final focusing in a driver 
have been completed and advanced simulations suggest that it will be possible to achieve adequate focusing at the required peak power. For example, (a) the high current experiment (HCX) [11] showed that a beam of driver current and brightness could be created, injected, and transported over short distances (short only because of limited funding). The source and injector created a $0.2-0.8 \mathrm{~A}, 1-2 \mathrm{MeV} \mathrm{K}^{+}$beam. A six quadrupole matching section preceded the periodic transport lattice of ten electrostatic quadrupoles. The periodic lattice period was $43 \mathrm{~cm}$, and the overall length of the matching and periodic focusing section was $5.7 \mathrm{~m}$. Though the beam area decreased from the emitter to the end of the matching section by about 1 order of magnitude, it was sufficiently well matched to maintain a normalized emittance $<1 \mathrm{~mm}$ mrad $(4 \mathrm{rms})$ despite the significant space-charge forces that in principle, if not well controlled, would ruin the emittance.

Scaled experiments and simulations addressed beam manipulations required in a driver. These scaled experiments used beams of $10-50 \mathrm{~mA}$ in order to test critical beam physics with the relevant perveance: (b) The stability boundary for the propagation of high space-charge beams over a wide range of tunes below a betatron phase advance of $\sigma_{o}=90^{\circ}$ per lattice period, was established in the single beam transport experiment (SBTE) with a space charge depressed tune as low as $20^{\circ}$. The experiment measured the evolution of the $200 \mathrm{keV} \mathrm{Cs}^{+}$beam phase space through 87 electrostatic quadrupoles [12]. (c) The transverse merging of four beams into a single quadrupole channel may be advantageous at the transition from electric quadrupole focusing to magnetic quadrupole focusing and the question of emittance growth due to beam-beam space-charge forces was important to address via experiment. This was demonstrated while retaining good beam quality in a scaled experiment [13-15]. A related application of transverse merging of beams is for the initial formation and injection of high-current beams. For some ion species it may be particularly advantageous to form the $\sim 1$ A beams by merging $>100$ very bright beamlets into the injector column [16] and is described in the next section. (d) Near the fusion target strong lenses are used to focus the beams to a few millimeters, limited mostly by the emittance of the beams. For each beam to undergo this significant reduction in size, the focusing system must have a relatively large aperture with low geometric aberrations. This was measured and modeled with and without the neutralization of the beam space charge with electron distributions in the scaled final focusing experiment [17], neutralized transport experiment [18], and with simultaneous longitudinal compression of the beam in the neutralized drift compression experiment (NDCX) [19,20]. (e) Experiments on bending and studying high spacecharge beams in circular machines are being explored with electrons at the University of Maryland Electron Ring (UMER) [21-23] and were studied with heavy ions in the recirculator experiment [24,25]. (f) Current amplification by longitudinal compression of a few to $\approx 50$ times has been demonstrated. The multiple beam experiment combined induction acceleration with a velocity ramp on a high space-charge $\mathrm{Cs}^{+}$beam. It showed little emittance growth while the line charge density of the beam increased by factor of a few, similar to that required in the driver [26]. Later, in NDCX, the addition of plasma neutralization in conjunction with a $\pm 15 \%$ velocity ramp furnished beams of a few nanoseconds duration, corresponding to 50-fold bunch compression and current amplification.

Particle-in-cell (PIC) codes for beam and beam-plasma interaction have both guided and been validated by comparison to the above experiments [11-30]. Computational tools were developed for the self-consistent modeling of the beams (and plasma if present) subject to the externally applied focusing field, acceleration field, self-field of the beam, as well as the fields from induced image-charge and currents. The WARP code [27-29] was specifically developed for heavy-ion IFE beam studies and has been used extensively for support of intense ion-beam experiments. The LSP code [30] employs a variety of plasma models and has also played an important role. The BEST program, featuring a nonlinear perturbative (" $\delta f$ ") approach, has been used to study beam oscillation modes [31].

In some of the experiments, electrons were intentionally introduced to beams to neutralize the space charge near the target, but for most of a driver, electrons are an unwanted presence in the beam line arising from ion-gas and ion-wall collisions. This presented a computational challenge due to the vastly different dynamical time scales between free electrons and ions. While the ability to carry out high resolution simulations has improved with the steadily increasing computing speeds, this time scale disparity stimulated the development of efficient algorithms for the WARP and POSINST codes to average over electron cyclotron periods while accurately modeling the influence of the electrons on the ion beam [32].

Simulations of radiation shielding of the superconducting magnets show a component lifetime exceeding 100 full-power years [33]. Thick-liquid wall chamber designs (e.g., HYLIFE-II) and related experiments suggest an attractive solution to first wall protection for IFE [6].

Nevertheless, the IRE is a very large step beyond the above experiments, and must integrate many of the challenges in a single research facility. Which outstanding scientific and technical questions ought to be answered before commencing IRE? (a) Drivers require $\sim 10^{14}$ ions/beam pulse to be focused and accelerated over long distances at $\sim 5-15 \mathrm{~Hz}$. This has not been demonstrated. How will gas buildup due to halo particle loss, secondary electrons (electron clouds), focusing field imperfections, and alignment tolerances influence the beam in the accelerator? Can these be adequately controlled? 
This can be answered with near-term experiments using a single beam with the beam energy and current characteristic of the front end of the IRE, at driver repetition rate in an accelerator long enough to allow several characteristic transverse oscillations of the space-charge-dominated beam and to assess the emittance growth for a driver-scale beam in the IRE. (b) What is the cost basis for the IRE and driver designs? Cost-effective injectors, quadrupole arrays, pulsers, insulators, and induction cells must be developed. This should be accompanied by collaborations with industry to develop and reduce the cost of custom accelerator components. Considerable work has been done in the past in these areas, but is incomplete to show readiness for a IRE. (c) A driver target chamber will use either an externally applied plasma to neutralize the beam, or the beam will become partially neutralized due to ionization of the background gas. Can this be adequately controlled at driver scale? The focusing elements should be sufficiently compact to be compatible with a liquid salt blanket, and to allow space for heat collection in the blanket, tritium breeding, etc. Near-term R\&D should yield an efficient, reliable, and durable focusing system design. This should be buttressed with relevant scaled beam experiments and continued development and application of advanced source-to-target modeling. Prototyping the key components of the IRE final-focus system and chamber might be desirable in the near term. (d) What target designs minimize driver cost and the beam focusing requirements? First, the most promising of the wide range of target design options that are compatible with HIF must be critically evaluated. The candidates include radiation driven indirect drive with a cylindrical hohlraum, hybrid direct/indirect designs, spherical and nonspherical direct-drive, fast ignition, and shock ignition. This should be followed by the design of specific IRE target experiments to answer the most important target design and physics questions.

These are among the most important scientific and technical questions for HIF. The answers, or at least significant progress toward the answers, would enable a credible physics design for IRE within 5-10 years. The research would reduce risk and would identify paths to costeffective components (e.g., injectors and acceleration modules) for IRE, and the DEMO. The research plan outlined below addresses these prerequisites on the road to an IRE or similar facility. It is advantageous to pursue these goals where valuable experiments are possible with existing equipment or accelerators.

The IRE will be an integrated demonstration of all the driver beam manipulations from source to target, including final focusing systems. The total energy in the beam pulse and pulse profile will enable energy deposition in targets well into the regime relevant to IFE implosions. If successful, it would demonstrate driver-scale creation of a high-current, low-emittance beam, driver repetition rate injection, matching and merging of multiple beams into the accelerator, acceleration and focusing through magnetic quadrupoles, longitudinal bunch control, and at the end of the accelerator, separation of beams, and compressing the beam from $\sim 100 \mathrm{~ns}$ to $\sim 10 \mathrm{~ns}$ at the target. The IRE accelerator physics includes all beam and plasma physics questions at a convincing scale, including emittance growth, halo formation, pulse compression, multiplebeam effects, and "beam loading" (the effects of the beam itself on the driving circuitry, and back onto the beam), and beam and plasma effects in and near the reactor chamber.

\section{ION SOURCES AND INJECTORS}

An accelerator facility requiring high reliability requires an ion source that is stable and rugged. In the first HIF workshops of the 1970s, the ion source was an immediate concern [34]. A survey of the state of the art found that: (1) Some ion sources produced currents in the microampere scale, at hundreds of $\mathrm{kV}$; other ion sources demonstrated many amperes but at only a few $\mathrm{kV}$ extraction voltage. (2) After the periodic table was surveyed for candidate ions, a few emerged as promising: The heavy alkali ions $(\mathrm{Cs}, \mathrm{Rb})$ were good candidates because of the ease of producing a single ionization state $(q=+1)$. Mercury is another ion that is easy to produce in a unique charge state, but exists naturally in a wide range of isotopes. Isotopic separation, a mature field, would add cost but may be acceptable. (3) At the time, ion thrusters produced beams for thousands of hours DC in a multiplebeam geometry with high current but relatively low voltage. This represents a highly developed sub-area of ion sources that overlaps with HIF in reliability and beam intensity. Xenon ion thrusters have been used on satellites recently [35].

Since then, HIF R\&D has demonstrated the production of single ion beams with the required emittance, current, and energy, suitable for injection into an induction linac $[36,37]$. The repetition rate for these sources was low, and the total operating hours and the number of extracted pulses were still well below the number of pulses required in a driver in $1 \mathrm{yr}$ at $\sim 10 \mathrm{~Hz}$. Studies carried out by the U.S. HIF research program include: (a) the $\mathrm{Cs}^{+1} 1 \mathrm{~A}$ source [38] demonstrated low emittance and high charge state and species purity in an ampere-scale $1 \mathrm{MeV}$ beam, created by a hot-plate ion emitter followed by an extraction electrode. Of order 100 such beams are needed in a fusion driver. (b) The 2 MV source and injector on the HCX experiment $[36,39]$ furnished a similar low-emittance $\mathrm{K}^{+}$ beam from a potassium aluminosilicate emitter for beam matching and transport experiments. (c) An alternative method is to create each of the required ampere-scale beams by merging many low current beamlets in an injector. This "multibeamlet" approach starts with a compact cluster of about 100 beamlets, each of order $\sim 1 \mathrm{~mm}$ radius and $<10 \mathrm{~mA}$, created by a plasma source. The beamlets are rapidly merged into a single beam during 
the extraction process. Such an injector (for argon ions) was tested (and shown to extrapolate well to xenon for a driver) $[37,39,40]$.

For a multiple-beam accelerator and target physics facility, R\&D demonstrating at least one design for a multiple-beam injector is a prerequisite. The demonstration should include two previously unverified features: creation and injection of at least several driver-scale beams, and successful operation at the driver repetition rate. Seven or nine beams might be considered a sufficient prototype of an injector that would ultimately require $\sim 100$ beams. There are no known fundamental problems here; rather, these are promising options to demonstrate an important aspect of a heavy-ion driver, not in use for other applications.

The merging and matching of many $\sim 1 \mathrm{~A}$ ion beams, created from sources that are wider than the unit cell size $(r \sim 0.01-0.2 \mathrm{~m})$ of a multiple-beam induction linac array, will require dipole as well as focusing fields to match the induction linac. The array of beams is steered and their envelopes matched to that required at the front end of the accelerator, where the overall transverse footprint is smaller (for the multibeamlet approach) or much smaller (for the monolithic hot-plate approach) than at the sources [16]. A physics design of the injector would be explored through advanced modeling of the beam and any background beam gas and halo-produced secondary electrons. The designs would first be verified on a test stand equivalent to the STS-500 [37] (a 500-kV ion source test stand furnishing flat high voltage (HV) pulses of up to $20-\mu \mathrm{s}$ duration) with one beam, to be followed by a multiplebeam test stand.

Each injector approach presents its own challenges: For example, in a large array of beams each sourced by many tiny (mm-size) plasma sources, can the gas load be kept low enough against emittance-increasing ion-gas collisions? For monolithic hot-plate sources each generating $\sim 1 \mathrm{~kW}$, can the attendant heat be managed effectively?

\section{INJECTION AND TRANSPORT OF A DRIVER-SCALE BEAM AT $\sim 10 \mathrm{~Hz}$}

Most heavy-ion fusion driver designs have gravitated toward a $5-15 \mathrm{~Hz}$ repetition rate. For a nominal $1-\mathrm{GWe}$ power plant, decreasing the rep rate leads to a requirement for bigger (higher yield) fusion targets, a bigger reactor chamber, and greater total driver beam energy. Increasing the repetition rate in the reactor is limited by gas condensation times and other relaxation times in the chamber. An ongoing question for operation of the accelerator is whether the pressure inside the beam lines, which is expected to rise immediately after a beam pulse, can be lowered fast enough for the next pulse or even be damaging to the pulse itself. An experiment aimed at quantifying and developing techniques to control the pressure rise, at least at the presently attainable beam energy and intensity, could be performed with existing equipment after modest modifications. This low-energy range is particularly relevant for HIF because the cross sections for ionization of background gas, ion stripping by background gas, and production of secondary electrons are all near their maxima or are relatively large at an ion kinetic energy of a few $\mathrm{MeV}$ [41].

The HCX injector [36] can be modified for experiments at $10 \mathrm{~Hz}$. The main modifications are to replace the two Marx charging power supplies with active turn-off power supplies rated for $8 \mathrm{~kW}$ and $0.25 \mathrm{~A}$. The charging path resistors may need to be decreased. This will enable operation up to $1.6 \mathrm{MV}$, with an initial pulse duration of $5 \mu \mathrm{s}$, and $0.5 \mathrm{~A} /$ pulse.

The possible experiments include gas-buildup and desorption studies in the injector, matching section, electrostatic transport section, and magnetic quadrupoles. Instrumented with fast pressure gauges to monitor the spatial and temporal evolution of the pressure, the experiment would have a beam tube with dimensions characteristic of a superconducting magnet bore to explore the efficacy of halo scrapers. Halo scrapers at normal incidence (expected to be better than grazing incidence) would be tested. The bore may be cryogenically cooled to explore the possibility of implementing cold bore superconducting magnet arrays.

In order to make more precise measurements [32] of the effect of electron clouds in magnetic quadrupoles, four new pulsed magnets, specially designed for low ion kinetic energy (short lattice period), can be added to the HCX beam line. A preliminary engineering design for the quadrupoles and experimental beam line includes diagnostic access, and induction acceleration gaps between the quadrupoles. The induction gaps are of interest; they will enable measurements of the degree to which they sweep electrons out of the beam in the gaps between quadrupoles.

A first round of electron cloud experiments on HCX showed emittance growth and distortions of phase space [32] though the electrons were mostly suppressed with biased electrodes. The interpretation of this result was confounded by the requirement to operate at a high betatron phase advance per lattice period $\left(\sigma_{o}>90^{\circ}\right)$, which introduces possible envelope control issues that are known to lead to emittance growth [42]. (The original magnets were designed as prototypes for the transport of much higher energy ions, so their geometry was not well suited for the $1-1.6 \mathrm{MeV} \mathrm{K}^{+}$ions.) The new magnet design and focusing gradient is designed specifically to enable measurements with $\sigma_{o} \leq 90^{\circ}$.

The principal outcome of the experiments would be an experimental verification of injection and transport of a $\sim 1$-ampere heavy ion beam at repetition rates required for IRE and a driver. PIC and gas-desorption models would be benchmarked by the experiments. 
There is, however, a need for experiments and modeling of driver-scale beams over a longer distance, as will be described in the following section.

\section{TRANSPORT THROUGH SEVERAL PLASMA (BEAM) OSCILLATIONS}

The previous section described experiments that can be carried out in a short term and rather inexpensively. This is because a short beam line enables answering key questions related to higher repetition rate operation. In this section, experiments are described that would probe emittance growth and longitudinal physics of such beams over a longer distance.

Transport and acceleration experiments over 40-100 quadrupoles would address driver beam physics questions such as transverse emittance growth of high space-charge beams over several plasma oscillations. Possible causative mechanisms are envelope mismatch, imperfect applied fields, electron clouds [43], and beam-gas interactions. Collective effects of space-charge waves are expected to relax after several plasma oscillations [44]. The phase advance for these oscillations per lattice period of length $L$ is approximately

$$
\sigma_{p} \approx \frac{L \sqrt{2 K}}{r_{b}},
$$

where $K$ is the generalized perveance,

$$
K=\frac{q \lambda}{2 \pi \epsilon_{o} \gamma^{3} \beta^{2} c^{2} m},
$$

$\lambda$ is the line charge density, and $r_{b}$ is the beam radius. For space-charge dominated beams the expression becomes:

$$
\sigma_{p} \approx \sqrt{2} \sigma_{o}
$$

Since the maximum of $\sigma_{o} \approx 80^{\circ}-85^{\circ}$, then $\sigma_{p} \leq$ $113^{\circ}-120^{\circ}$. Halo formation and control via periodic collimation would also be explored in such an experiment.

The SBTE $\mathrm{Cs}^{+}$beam current was low compared to a driver, but the perveance was characteristic of a driver. The transport channel had a large aperture compared to the rms beam size and the experiments primarily focused on transverse dynamics and stability issues in the middle of the bunch.

Each beam bunch has a mostly constant current except for the ends. Thus, the longitudinal space-charge forces are very small, except at the ends of the bunch, where the longitudinal self-field is proportional to the gradient of the line charge density, $d \lambda / d z$.

It is appropriate to note characteristic longitudinal time scales, which are much longer than the transverse dynamics time scales described above. Because of the high ion mass, long bunch length, and low longitudinal emittance (on the order of $10^{-2} \mathrm{eV} \mathrm{sec}$ ), single ions move very slowly within the bunch, the analog to synchrotron motion in an rf accelerator. In fact, a particle will only traverse a small fraction of the bunch length in the whole accelerator. But space-charge waves move at a greater speed, $\sim 0.1-0.2 \mathrm{~m} / \mu \mathrm{s}$, and will traverse a 5-10 $\mathrm{m}$ bunch a few times in the accelerator.

Limiting the dilution of longitudinal phase space is essential to achieving the short pulses required for target experiments in IRE and a driver. Thus, experiments to detect and control the seeds of longitudinal emittance growth due to waveform imperfections and temperature anisotropy (longitudinal/transverse) will address theoretical predictions such as in Ref. [45].

The result would be a single-beam test with full-scale beam current and emittance using accelerator components characteristic of the front end of the IRE. The physics objectives are closely related to those identified in previous accelerator design concepts $[3,46]$, and the development plan outlined at the 2002 Snowmass Fusion Summer Study [47]. The beam current and top kinetic energy- $0.5 \mathrm{~A}$ at injection and $10 \mathrm{MeV}$ final kinetic energy was proposed at the time-depends on the available resources, technology limits, and detailed experimental goals. As noted above, electron accumulation would be inhibited due to the efficient removal of electrons by accelerating gap fields between quadrupoles. This can be studied experimentally with a limited number of acceleration modules. Though it is valuable to study each of these effects in isolation, eventually a more convincing proof of principle for IRE is an experiment that integrates all of the effects of sequential beam manipulations with imperfections in their applications.

These experiments that precede the IRE are most valuable if the accelerator architecture resembles the IRE and an HIF driver. Technology R\&D is critical for creating novel components that demonstrate the needed technical specifications (acceleration gradient, focusing strength) and fabrication techniques at an acceptable cost.

In addition to single-beam experiments, studies are required to clarify the interactions (longitudinal and transverse) between the multiple beams that thread each induction core. Transverse deflections, and inductive effects

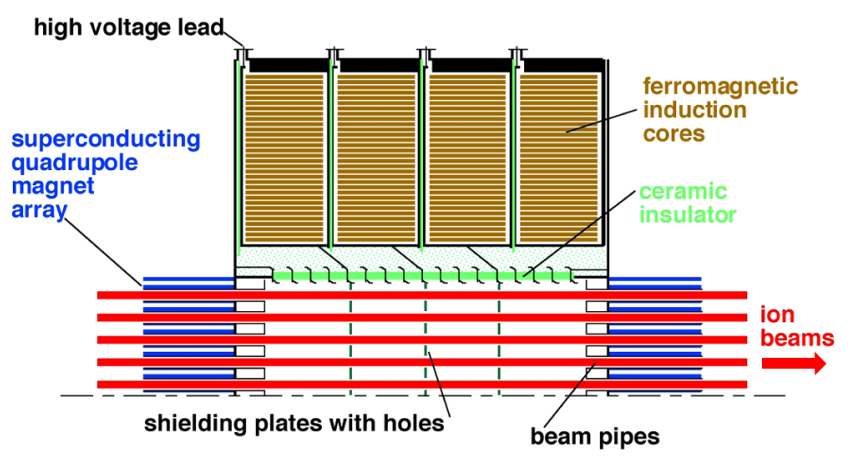

FIG. 2. Schematic of one-half of a lattice period in a multibeam induction linac. 
associated with the fact that the magnetic fields generated by all the beams are additive, need to be understood. This can be pursued by a combination of scaled models (e.g., electron beams), measurements of the key impedances, and advanced simulations. See Fig. 2.

The understanding from these experiments may also be helpful to high-intensity hadron accelerators for applications such as neutron spallation sources.

\section{DRIFT COMPRESSION, BENDING, AND FINAL FOCUS}

At the end of the accelerator, the beam must be compressed to the short length required at the target. Immediately preceding the target, final focusing and dipole magnets focus and aim the many beams onto the target with the required illumination geometry. To allow for the use of liquid-wall protection for most of the target chamber structural wall, the targets are usually designed to accommodate groups of beams in a compact bundle (two groups as in, e.g., [6]; but one group might be feasible). In some designs the velocity ramp on the beams is removed due to longitudinal space-charge repulsion, thus eliminating the need to accommodate a large momentum spread in the final focusing elements. In other designs, the beam passes through plasma that cancels the beam space-charge field, enabling shorter beam bunches or lower kinetic energy, and higher perveance ion beams. The plasma electron density must exceed that of the ion beam. Near the target, the ion-beam density may be $10^{14} / \mathrm{cm}^{3}$, depending on the driver design.

The standoff from the last lens to the target is 5-10 meters, to contain the energy released by the fusion reactions and the neutron absorbing liquid jets. To achieve the required spot size, a suitable convergence angle, $\theta$, must be chosen, resulting in a beam size of $\sim 10 \mathrm{~cm}$ at the last focusing magnet, considerably larger than in the accelerator.

For an ideal focusing system and relatively low beam current, focusing is limited only by the emittance of the beam:

$$
r \approx \frac{\epsilon}{\theta},
$$

where $\varepsilon$ is the unnormalized emittance, and $r$ is the minimum spot size. However, other factors that will increase the spot size are alignment, space charge, chromatic aberrations, and geometric aberrations. Contributing approximately in quadrature, they are described below.

Though steering and aiming of charged particle beams can be very precise in principle, there is a phase or coherence problem that arises in this application, the exact solution to which usually requires challenging timedependent correction elements. In the accelerator and, in particular, during the initial compression of the bunch, different parts of the beam bunch will have slightly different kinetic energy and betatron phase advance. In the thin lens approximation:

$$
\sigma_{o} \approx \eta \kappa\left(\frac{L}{2}\right)^{2}
$$

where $L$ is the quadrupole lattice period, $\eta$ is the quadrupole occupancy, and $\kappa$ is the focusing parameter appearing in the rms envelope equations. For magnetic quadrupoles,

$$
\kappa=\frac{G(z)}{[B \rho]},
$$

where $G(z)$ is the transverse gradient of the magnetic field.

In combination with small alignment errors leading to centroid oscillations in the lattice, different slices of the beam will have centroid motion that is out of phase with the rest of the beam, leading to an effective smearing of the beam profile at the target.

The generalized perveance decreases inversely as $\gamma^{3} \beta^{2}$ leading to easier focusing at higher kinetic energy. For target designs requiring a focal spot of a few $\mathrm{mm}$ or more, the contribution of space charge to the spot size usually is relatively small above ion kinetic energy of $7-10 \mathrm{GeV}$. Some neutralization is inevitable as the beam traverses several meters to the target. This is due to the liberation of electrons from beam-gas collisions, and the photoionization of the beam from $\mathrm{x}$ rays emanating from the target.

Focusing systems with static fields have been designed to be sufficiently achromatic for momentum spreads of $\Delta p / p<1 \%$. The momentum spread is determined by the longitudinal emittance of the beam, and also whether the head-to-tail velocity ramp (which compresses the beam bunch, $\Delta p / p \sim 3 \%$ ) is mostly removed by the stagnation of the bunch length due to longitudinal space-charge forces, or if that force is removed and the bunch is further shortened by the introduction of a neutralizing plasma or other source of electrons.

The contribution of geometric aberrations scale as $\theta^{3}$, which, combined with the other focusing constraints discussed here, has limited the convergence angle of the beam at the exit of the final lens to $\theta \approx 10-20$ milliradians.

Regarding the radiation environment near the target chamber, the magnets are superconducting and the coils must be protected from radiation flux originating from the fusion target.

Radiation flux through the beam line could lead to superconducting coil quenching and degradation. It can be controlled with a combination of shielding structures near the coils, and radiation dumps can limit deleterious effects upstream when combined with dipole bends in the beam line [33]. Managing the radiation flux differs from laser IFE, which is less compatible with liquid-wall protection of the first wall, and where the final optics are necessarily in the line of sight of the flux of neutrons, charged particles and photons emitted by the fusion target.

The steering of beams through large angles to correctly illuminate the target raises questions of chromatic dispersion of the beam bunch. Regarding space-charge effects, 
the bending of beam lines breaks the symmetry of a straight beam line, leading to asymmetric fields from the image-charge effect in the conducting vacuum chamber walls. Past simulations have shown these effects to be benign within parameter ranges compatible with the driver and target requirements [48-51]. These questions should be revisited with simulations in much greater detail. The results may lead to the design of a relevant experiment possible at low ion kinetic energy that would significantly reduce risk for the IRE.

The focusing system (and accelerator) design goals are greatly affected by the required focal spot size and bunch duration. For example, for two target designs requiring either $0.3-\mathrm{mm}$ or $3-\mathrm{mm}$ focal spot radius, and a bunch duration of 0.2 or $10 \mathrm{~ns}$, there is usually a great impact on the focusing system design, and on the transverse and longitudinal emittance of the beam. This is to be expected, since at the extrema of this example, the six-dimensional phase-space density differs by a factor of 500 .

As the driver beam compresses in the most common driver designs, space charge removes much of the beam's head-to-tail velocity ramp. This removal of a velocity ramp (or stagnation of the inward flow, as viewed in the beam frame) renders the beam nearly monoenergetic, minimizing the chromatic aberration that would otherwise broaden the focal spot on target. NDCX-II will be used to study stagnation with an experiment to diagnose the beam at intermediate stations (measure 6D phase space), and then possibly position a final-focus magnet at the stagnation point (the location of which can be adjusted by tuning the amplitudes of the applied accelerating voltages). This is another example of an HIF experiment that can be accomplished with straightforward modifications of the beam line.

On NDCX-II, the generation of precision voltage pulses across induction-cell gaps is necessary for the warm-dense matter mission, so the experience gained will carry over directly to designing larger machines. For example, a varied set of experiments may be carried out to explore some of the manipulations that may be employed to shape the pulse of individual driver beams.

\section{TARGET PHYSICS}

The fusion power DEMO mentioned at the beginning of this paper will demonstrate high rep-rate operation at low fusion yield (of order $30 \mathrm{MJ}$ ) and may include exploration of higher gain and yield targets. For the IRE to validate the beam-target interaction physics, one would like the beamplasma frequency and target conditions to be close to driver values. How close is a matter of judgment and involves tradeoffs between cost and performance. Validating beamtarget interaction physics in the IRE at $T>50 \mathrm{eV}$ or greater requires a flux greater than $\sim 3 \times 10^{12} \mathrm{~W} / \mathrm{cm}^{2}$ and multi-kJ in the pulse. Reference [4] showed beam parameters required to achieve these goals. Experiments beyond this basic goal depend on the HIF driver target designs considered, as described below.

At the beginning of the driver, the beams are injected with a large safety factor in phase-space density compared to the requirement of the fusion target. A considerable fraction of this phase-space density margin will be diluted in the acceleration and compression of the beam, and the degree is to be determined via experiment and modeling. This margin must be incorporated in the target design, and kept conservative until a body of evidence supports reducing the safety factor.

Heavy ion targets can be characterized in a multidimensional parameter space [52], with any target having three continuously varying parameters: (1) degree of direct drive (whereby the ion beams directly heat the outer layers of the fuel capsule) vs indirect drive (whereby the ion beams heat a cylindrical "hohlraum" that radiates x rays that, in turn, heat the capsule); (2) scale of target; and (3) the mode of ignition (where hot-spot ignition requires the highest value of the central adiabat just before ignition, and fast ignition requires a low central adiabat, with shock ignition somewhere in between.) In hot-spot ignition, the gaseous fuel contained within a dense shell is heated as the fuel shell is compressed in radius and thickness. In fast ignition, the fuel is first compressed, followed by a separate "ignition" pulse that raises the temperature of the compressed fuel. These key parameters are described below: As one goes from indirect drive to direct drive, one finds better coupling but harder alignment and beam smoothness requirements. As one increases the target scale and focal spot requirement of the target (keeping the type of drive and mode of ignition fixed), beam phase-space requirements are eased and there is a potential for higher gain, and lower repetition rate, but the driver energy requirement increases. As one proceeds from hot-spot ignition to fast ignition, the pulse duration and spot radius decreases, increasing the phasespace density requirements or increasing ion energy, but the compressibility also decreases, favoring more stable targets.

For direct drive, the energy is usually deposited in the outer layer (sometimes a dense material) and in other designs, within the outer fuel layer (Fig. 3). For indirect drive hohlraum targets, the symmetry in the illumination of the fuel is controlled in laser targets by the shape and composition of the hohlraum wall. In laser IFE hohlraums, there is an entrance hole for the laser beams, and the laser beams are aimed at the inner cylindrical wall of the hohlraum. For heavy-ion targets, the asymmetries are controlled by the composition and placement of shields and converters in the path of the beam (instead of entrance holes) and within the hohlraum.

Ideally, the IRE would be flexible enough to study issues such as stability and uniformity for a wide class of targets. Indirectly driven targets are an attractive approach for HIF because they allow two-sided illumination, an illumination 

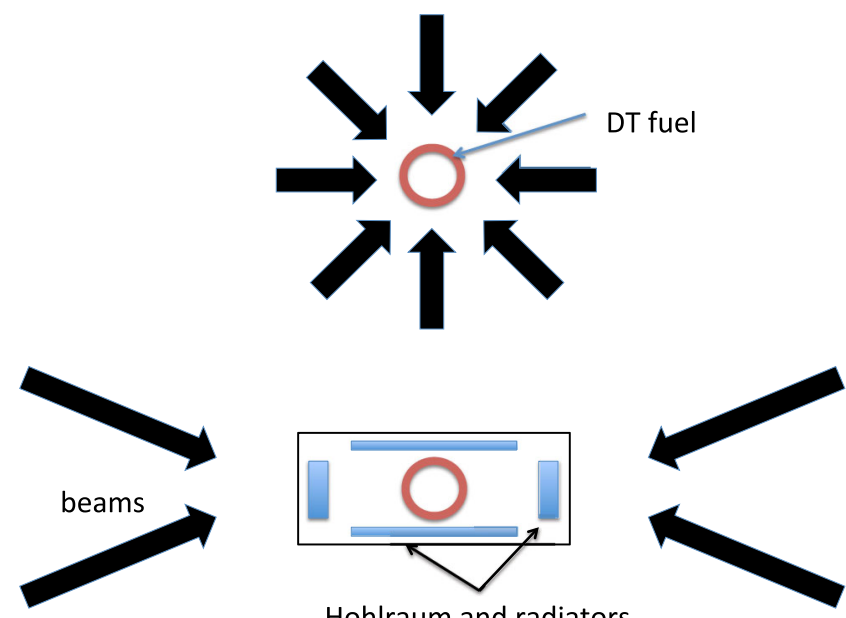

Hohlraum and radiators

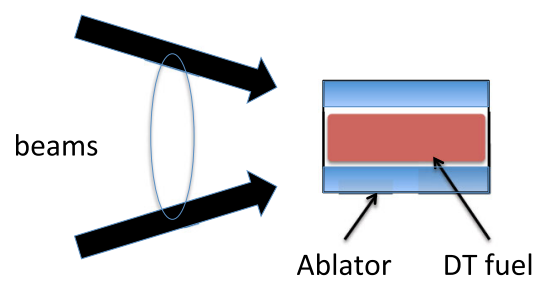

FIG. 3. Three types of targets are shown to illustrate the beam configurations for spherical direct illumination (top), two-sided illumination (middle), and one-sided cylindrically symmetric direct drive (bottom). Possible variations in illumination geometry among these cases are discussed in the text.

geometry that is compatible with thick-liquid wall protected chambers. Moreover, much of the target physics will be established on the NIF and elsewhere. Other target options such as direct drive, or aspherical direct drive, may offer higher target gain and/or lower driver energy.

Determining the cost and target physics capabilities of the IRE is a principal goal of target R\&D within the next several years. The IRE target physics experiments should answer questions unique to heavy-ion-driven targets and then lead to confident DEMO target and accelerator designs. Near-term surrogate experiments might be done (e.g. on NIF, OMEGA, etc.) to elucidate specific heavy-ion fusion target physics topics. At this point none have been identified as prerequisites for an IRE, but this would be explored further as part of the IRE and driver target design effort.

Much is known about ion energy deposition in cold matter. It is known to be different in hot, dense matter, leading to possibly favorable changes in the ion range. Some experiments have validated and observed range shortening in low-density plasmas [53]. More accurate modeling in the IFE temperature and density regime will require an experimental campaign at IRE.

Implosion experiments on IRE would require a more complex final focusing system (vs planar target experiments) to achieve the needed target illumination geometry.
However, indirect drive with two-sided illumination (or the single-sided X-target-see below), in principle allows clusters of beams with reduced solid angle compared to the uniform illumination required of spherical targets. Polar direct-drive versions of spherical targets also allow smaller solid angle of the beam array. Single-sided illumination of both indirect and direct-drive targets is possible in principle but significant target (and beam focusing) modeling for these concepts would be needed to develop a design for IRE experiments.

Three example designs in the target parameter space are described below, with related challenges and possible improvements: (1) cylindrical hohlraum indirect drive targets; (2) spherical targets; (3) cylindrically symmetric direct-drive targets, including the X-target.

1. Cylindrical hohlraum, indirect drive targets.Detailed designs include the "distributed radiator" targets [54,55], and their close relatives the "closely coupled target" [54,56] and the "hybrid" target [57]. These are the most mature HIF target designs, with integrated 2D models having gains ranging from $60-130$ for 3-7 MJ input energy. Specific, and relatively detailed compatible accelerator designs, with considerable safety margins in the beam parameters have also been published for this class of targets.

Much is being learned about hohlraum targets as part of the National Ignition Campaign at NIF. Important physics details of radiation dominated indirect drive are coming to light, including issues of sensitivity to beam uniformity on the hohlraum and time-dependent symmetry. Implosion physics is also being tested, with issues related to the Rayleigh Taylor instability, symmetry, the mixing of one target material with another and capsule roughness receiving scrutiny. Results of these experiments will have direct relevance to a variety of HIF target designs.

For example, in both laser-driven and ion-driven indirect drive, the fuel capsule is radiation driven, so the capsule implosion and burn physics are the same. This is especially true if the heavy ion hohlraum and laser hohlraum are heated to the same radiation temperature.

There are differences in the hohlraum design and in the energy deposition between laser and heavy ion indirect targets: In the laser targets, there are holes in the targets to allow the laser light to enter, and the laser-plasma interaction must be manageable. Also, the physical mechanism of beam energy deposition is more volumetric in HIF.

The heavy ion hohlraum target designs and beam power profiles were derived by demanding the same temperature versus time profile in the hohlraum as were developed for laser hohlraum targets. This might have forced some beam power (versus time) features and constraints that are not fundamental requirements. The accelerator cost and design optimization was subordinate to the specified pulse shape on the target. This should be revisited with a more global optimization of the driver design and cost. 
The target design for the Robust Point Design driver [55] calls for an accelerator with two values of ion kinetic energy, in separate beam lines. It appears that a single-energy-target-preferred for the accelerator design-could be designed at some energy or yield penalty. (Maintaining time-dependent symmetry is the challenge, as target heating changes ion range over the course of the pulse.)

The hybrid target [57], attractive because it allows for larger beam radii on target ( $>5 \mathrm{~mm})$, has two noteworthy unresolved issues: (i) The original design has a small $\left(\sim 10^{\circ}\right)$ acceptance cone angle for the bundle of beams, which was challenging for the final focusing optics design. If the cone angle could be increased to be consistent with a $20^{\circ}$ bundle of beams on each side of the target, the design of the final focusing optics would be much less constrained. (ii) The design was less robust than the distributed radiator. The distributed radiator target and close-coupled targets are designed for a larger cone angle, and they are more numerically robust than hybrid targets.

Compared to spherical targets (below) hohlraum targets are more complicated to fabricate, though considerable headway has been made in conceptual designs of fabrication facilities that appear to yield low cost, high precision, and reliable targets [58].

2. Spherical targets. - In spherical targets, the ion-beam converters are spherically distributed around the DT fuel [59-64]. A tamper (using high density material) may surround the capsule and increases the coupling efficiency. However, the tamper also absorbs beam energy before the ions reach the converter, offsetting some of the increased coupling efficiency. The tamper allows ions of higher range to illuminate the target, easing beam phase space and perveance requirements on the accelerator. Since the targets are layered spheres they are relatively simple to fabricate. They have high gain and have been designed with single ion-kinetic-energies $(\sim 2-10 \mathrm{GeV})$. Stability studies of tamped direct-drive targets are currently under way [65]. For tamped spherical targets with shock ignition, further study is required to determine if shock ignition is compatible with a single ion kinetic energy.

3. Cylindrically symmetric direct-drive targets, and the $X$-target.-A cylindrical, fast-ignition target has been designed with one-sided illumination geometry [66]. The cylindrical geometry lowers the gain (compared to spherical), but the high-gain potential of fast ignition compensates for the effect of geometry. They require a higher ion range and so can accommodate high ion kinetic energies. The fast-ignition pulse requires high power, and a small focal spot, which in turn demands a higher phase-space density in the accelerator. The driver concept for this target is at this point immature. The needed beam spot radius is $\sim 0.05 \mathrm{~mm}$, and the ignition pulse duration is $\sim 0.2 \mathrm{~ns}$.

The X-target [67] is named for the outer case geometry (a metallic tamper) arranged approximately as a surface of revolution. It is a solid target with quasispherical symmetry, but with two cones removed from the sphere. The case is filled with outer shells of "propellant" such as aluminum, with DT fuel interior to the propellant. The ion beams illuminate the target from one side only, deposit their energy volumetrically in the propellant or the DT, and assemble fuel with a sequence of two quasispherical

TABLE I. Beam requirements for example target designs.

\begin{tabular}{|c|c|c|c|c|c|c|c|c|c|c|}
\hline \multirow[t]{2}{*}{$\begin{array}{l}\text { Target design } \\
\text { and citation }\end{array}$} & \multicolumn{2}{|c|}{$\begin{array}{l}\text { Cylindrical hohlraum, } \\
\text { indirect drive: } \\
\text { "Hybrid" [57] }\end{array}$} & \multicolumn{2}{|c|}{$\begin{array}{l}\text { Cylindrical hohlraum, } \\
\text { indirect drive: } \\
\text { "Distributed radiator } \\
\text { (RPD)" [57] }\end{array}$} & \multicolumn{2}{|c|}{$\begin{array}{c}\text { Spherical: } \\
\text { "Tamped } \\
\text { direct drive" } \\
{[65]}\end{array}$} & \multicolumn{2}{|c|}{ X-Target [67] } & \multicolumn{2}{|c|}{$\begin{array}{l}\text { Cylindrically } \\
\text { symmetric } \\
\text { direct drive, fast } \\
\text { Ignition [66] }\end{array}$} \\
\hline & Foot & Main & Foot & Main & Foot & Main & Compressor & Ignitor & Compressor & Ignitor \\
\hline $\begin{array}{l}\text { Energy (in pulse) } \\
\text { (MJ) }\end{array}$ & 1.7 & 5 & 1.76 & 5.25 & 0.2 & 0.45 & 2 & 3 & 7.1 & 0.4 \\
\hline Pulse duration (ns) & 9.7 & 8.3 & 19.6 & 10.3 & 23 & 7 & 2 & 0.2 & 75 & 0.2 \\
\hline $\begin{array}{l}\text { Beam radius, or } \\
\text { semiminor } \\
\times \text { major radii } \\
\text { for elliptical } \\
\text { spots }(\mathrm{mm})\end{array}$ & $3.8 \times 5.4$ & $3.8 \times 5.4$ & $2.3 \times 4.2$ & $1.8 \times 4.2$ & 1 & 1 & 0.425 & 0.255 & 0.5 & 0.05 \\
\hline Ion range $\left(\mathrm{g} / \mathrm{cm}^{2}\right)$ & 0.031 & 0.049 & 0.034 & 0.042 & 0.038 & 0.038 & 2 & 2 & 4 & 4 \\
\hline $\begin{array}{l}\text { Illumination } \\
\text { geometry }\end{array}$ & Two & ided & Two & ided & $\mathrm{Sph}$ & rical & One-sic & & Two-sid & \\
\hline Target gain & & & & & & & 300 & & 100 & \\
\hline Ion mass (amu) & 208 & 208 & 208 & 208 & 207 & 207 & 238 & 238 & 207 & 207 \\
\hline $\begin{array}{l}\text { Ion kinetic } \\
\text { energy }(\mathrm{GeV})\end{array}$ & 3 & 4.5 & 3.3 & 4 & 3.7 & 3.7 & 90 & 90 & 100 & 100 \\
\hline Charge in pulse (mC) & 0.57 & 1.11 & 0.53 & 1.31 & 0.05 & 0.12 & 0.02 & 0.03 & 0.07 & 0.00 \\
\hline Current in pulse) (kA) & 58.4 & 133.9 & 27.2 & 127.4 & 2.4 & 17.4 & 11.1 & 166.7 & 0.9 & 20.0 \\
\hline
\end{tabular}


shocks. A final short igniter pulse provides the spark to ignite the assembled DT fuel. X-targets are inherently one-sided drive and have high coupling efficiencies, reduced RT stability issues associated with the low compression ratio, and a potential for high yields $(\sim \mathrm{GJ})$ and high gains. Kelvin Helmholtz $(\mathrm{KH})$ instability associated with the quasispherical flow along the material boundaries is still under investigation. The high gains require the creation of high densities under the quasi-3D compression. The target requires a high range ion and thus higher ion kinetic energies. High power and small focal spot beams are needed for fast ignition. The driver concepts for these schemes are, at this point, immature. The beam spot radius for the igniter beam is $\sim 0.2 \mathrm{~mm}$, and the ignition pulse duration is $\sim 0.2 \mathrm{~ns}$. For the $\mathrm{X}$-target (and the cylindrical target fast-ignition target), a single ion energy for the target was chosen. The IRE could explore issues associated with $\mathrm{KH}$ instability, and fuel assembly.

Table I summarizes the beam requirements for some of the specific target designs cited above. Each target's requirements are given by the top five rows in Table I, and are in principle compatible with a range of values of customary beam parameters (ion mass, kinetic energy, and beam current) provided that the energy in each pulse, the beam radius on the target, and the ion range are satisfied. That is, the last four rows (ion mass, energy, charge, and current in pulse) are subordinate to the target requirements above them. As stated earlier, the required beam energy per pulse varies among target designs by a factor of several, which will influence the number of parallel beams and other aspects of the accelerator design. The designs vary in their sensitivity to instabilities during the compression and heating of the fuel, and in turn to the precision in steering, the transverse beam profile, and the beam power temporal profile.

In summary, near-term research would be centered on the development of a suite of IRE IFE target physics experiments, closely tied to robust HIF target designs for a DEMO. The goal is to design a flexible IRE that will allow critical scientific questions to be answered for more confident as well as speculative target designs.

\section{TECHNOLOGY DEVELOPMENT}

IFE systems codes combine various constraints set by beam physics (such as transportable current for a given focusing field and aperture), technology (e.g., maximum achievable acceleration gradient), and component cost. The constraints are sometimes set by significant extrapolations, introducing large uncertainties in the optimization of the complex driver system for cost and performance. In order to reduce this uncertainty, $R \& D$ on heretoforeuntested and novel HIF accelerator components is needed. This is a normal and necessary aspect of accelerator $R \& D$ in preparation for the next big step (viz., several years of
$\mathrm{R} \& \mathrm{D}$ in preparation for, and before setting the final design specifications for, very large accelerators around the world, such as RHIC at Brookhaven National Laboratory, SNS at Oak Ridge National Laboratory, and the LHC at CERN.). This will enable the systems studies to go beyond sensitivity analyses to actual optimization and design. Two examples aimed at the exploration of the novel accelerator architectures and for reducing the risk for IRE are described below.

\section{A. Example 1: High-gradient accelerator modules}

System studies show cost reductions for higher gradients but data for such extrapolations are scarce. Hovingh et al. [5] studied the dependence of the driver cost and efficiency on the surface flashover field limit, and found that doubling the flashover limit decreased the cost of the driver by $24 \%$ and increased the efficiency by $11 \%$. The accelerator footprint is nearly inversely proportional to the flashover limit and directly impacts geographic siting choices.

The repeat structure in the accelerator is the half-period $L / 2$, which has a quadrupole of length $\eta \cdot L / 2$, and an accelerating column of length $(1-\eta) \cdot L / 2$. The "occupancy," $\eta$, varies from about 0.8 at the beginning of the accelerator to about $0.1-0.2$ over much of the accelerator. To obtain an average acceleration rate $\hat{E}$, the column must provide $E_{c}=\hat{E} /(1-\eta)$. The transverse dimension of the multiple-beam focusing array is about $1 \mathrm{~m}$. The inner diameter of the induction cores is slightly greater than this diameter.

For most of the accelerator, the voltage increment between quadrupoles is a few MV, or whatever voltage holding and design choices allow. The acceleration gap designs usually include several plates electrically connected to the column for grading, with multiple apertures through which the numerous beams pass. In the systems studies, the gradient has been about $1 \mathrm{MV} /$ meter. The basis for this limit has been the somewhat less than $1-\mathrm{MV} /$ meter gradient in electron beam induction linacs, and also the scarcity of relevant experimental data on column HV holding above $\approx 1 \mathrm{MV}$. In electron induction accelerators, there was no need to strive for higher acceleration rates because the transport focusing costs were low. In contrast, the transport costs in HIF drivers are comparable to the acceleration modules.

To raise the column voltage holding limit $\left[V_{c}=\right.$ $\left.E_{c} \cdot(1-\eta) \cdot L / 2\right]$ subdividing the insulator into $N$ sections raises the voltage holding approximately as $N^{1 / 2}$, to increase voltage holding along a column insulator, and likewise, by using more metal partitions within the vacuum regions, raises the vacuum breakdown limit. Ordinarily, this insulator surface flashover has been the weak link. If, by a combination of axial and radial subdivisions, the surface breakdown is increased sufficiently, then vacuum breakdown between metal surfaces along the middle of the column would become the limit. 
Van-de-Graaf accelerators achieve several MV of acceleration along insulating columns, with $1 \leq E_{c} \leq$ $1.7 \mathrm{MV} /$ meter being a comfortable operating range [68]. It is important to note that they tend to have small apertures, with considerable investment into numerous baffles and electrodes along segmented columns, and low magnetic fields to deflect low energy stray charged particles and to prevent acceleration of back-streaming electrons to high energy. The $2 \mathrm{MV}$ injector [36] which serves as the front end for the HCX, has an insulating column length of 2.4 meters, implying an equivalent $\hat{E} \approx$ $0.83 \mathrm{MV} / \mathrm{m}$. The Dual-Axis Radiographic Hydrodynamic Test facility (DARHT-II) electron beam injector has a peak voltage of $3.2 \mathrm{MV}$ held across a $4.4 \mathrm{~m}$ insulating column [69].

Some approaches that might lead to increased gradient for HIF drivers include the implementation of radial insulators (vs insulating columns entirely parallel to the beam line), and the utilization of high-gradient insulators based upon very thin layers of graded columns [70].

An example of a possible near-term experiment with a modest investment is a test stand enabling high-gradient experiments of various acceleration gap geometries. This can be accomplished using spare advanced test accelerator (ATA) cells [71], which may also be used for a possible upgrade to NDCX-II. The ATA induction accelerator created a $10-\mathrm{kA}, 50-\mathrm{MeV}$ electron beam with a 200 induction cells operating at $5 \mathrm{~Hz}$, with burst mode capability up to $1 \mathrm{kHz}$. The ferrite loaded ATA cells have a 0.23-m inner diameter, smaller than the size of the multibeam test columns, therefore it becomes necessary to add the voltages of the cells on either side with a metal rod, as was done in previous induction linacs to furnish a high voltage pulse to an injector (e.g., RTA injector [72]). The accelerating gap and column itself should include the principal features of a driver: plates with multiple beam-hole apertures connected to the insulating column, and provisions for exploring baffle geometries. The experiment would not transport a beam or multiple beams. It would, however, provide valuable and presently unknown limits on $\hat{E}$, which vary in systems codes from 1 to $3 \mathrm{MV} / \mathrm{m}$. Using 16 ATA modules, operating at $250 \mathrm{kV}$ each, a total gap voltage of $4 \mathrm{MV}$ in a $70 \mathrm{~ns}$ pulse could be established. This is relatively close to the 100-200 ns pulse in most of the driver. Figure 4 shows a conceptual sketch showing a one-meter prototype HIF accelerating column sandwiched between a much lower insulating column and the ATA induction cores. As a preliminary experimental configuration, a test stand for a column of approximately half the one-meter column shown would require half as many induction cores to establish the same $E_{c}$.

Because there is no beam loading, several cells can be driven by one Blumlein pulser. X-ray diagnostics would indicate incipient breakdown, and an X-ray pinhole camera could isolate weak points in the system. The absence of beam also means that the measured voltage limits would be derated for use with beam, to compensate for the lower voltage holding caused by stray beam particles (halo) striking the accelerator structure. But even without beam, the voltage holding limits for the high cumulative voltages along one insulator for pulses of the required short duration would yield valuable new information.

\section{B. Example 2: Magnet array prototyping and testing}

There are two main types of magnets for a driver: focusing quadrupoles in the accelerator and final focusing magnets near the target chamber. For beam physics, engineering and economic reasons they are superconducting. Dipoles or displaced quadrupoles for bending are a minor concern.
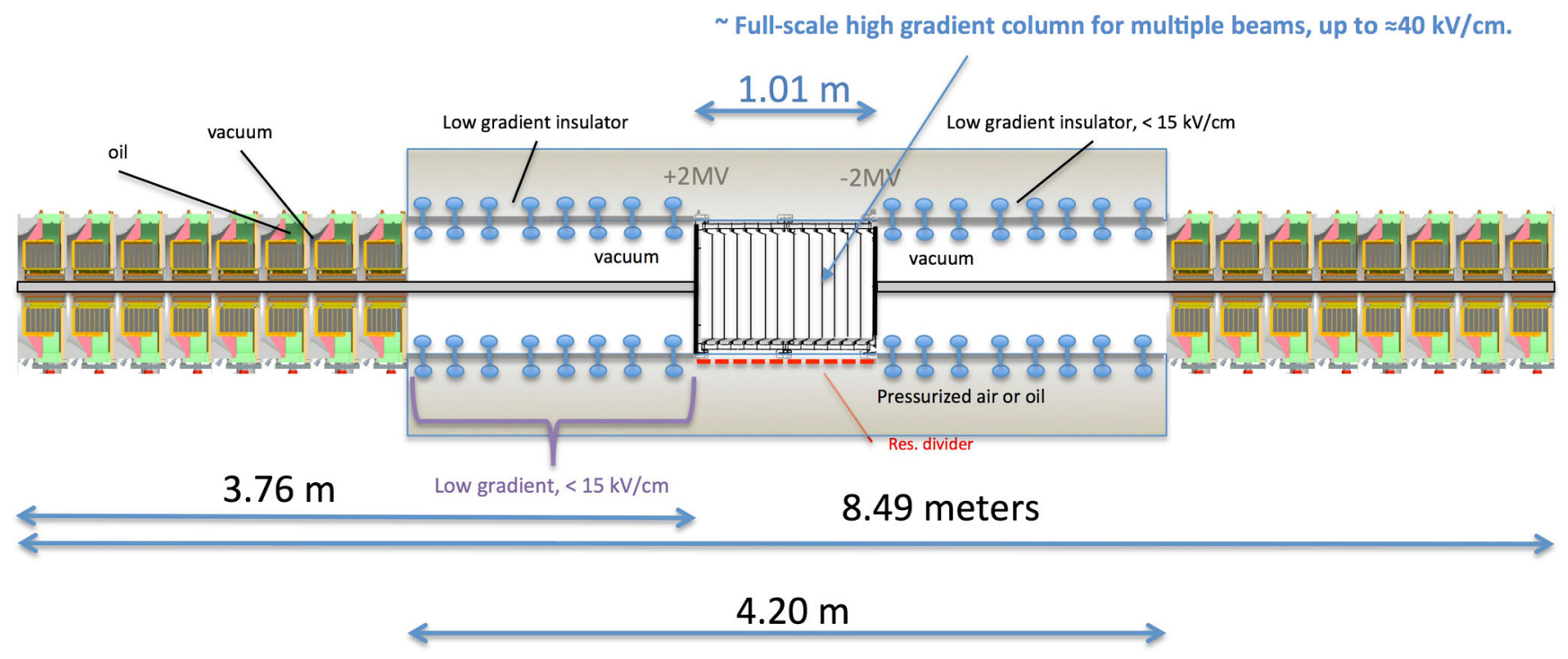

FIG. 4. Concept for a high-gradient HIF column test stand driven by a series of $250-\mathrm{kV}$ modules. The combined voltage across the test column could be up to $\Delta \mathrm{V} \approx 4 \mathrm{MV}$, as much as required in many conceptual drivers. 
This focusing array in the accelerator has unique challenges compared to high-energy physics accelerator magnets in compactness, coil layout, edge termination, alignment, cryogenics, and vacuum [73]. A close-packed array of superconducting quadrupoles sharing flux in a common cryostat has never been constructed, and these transport magnet arrays comprise $1 / 4$ to $1 / 3$ of the cost of the driver. An R\&D design and testing effort led to a superconducting quadrupole array design for HIF accelerators and then for a single-beam magnet design maintaining the key features on which the array design was based (see Fig. 5) [74]. The focusing set the lattice spacing required for a low-energy $(1.6-10 \mathrm{MeV})$, high line charge density $(\lambda \approx 0.2 \mu \mathrm{C} / \mathrm{m}) \mathrm{K}^{+}$beam. The design optimization after the first prototype magnets led to a "block-coil" (square) geometry with eight doublepancake coils with square ends. The later prototype tests showed a conductor limited (SSC Ni-Ti) gradient $(132 \mathrm{~T} / \mathrm{m})$ after two quenches. There was no magnet retraining required after thermal cycles and no significant dependence on ramp rate up to $200 \mathrm{~A} / \mathrm{sec}$. The short-sample current limit was 3000 A. A multipole decomposition of the magnetic field quality agreed with model predictions up to the 20-pole. The R\&D ended in 2004 with the production and successful testing of a prototype quadrupole doublet in a compact cryostat suitable for transport and acceleration of intense beams of $(\lambda \approx 0.2 \mu \mathrm{C} / \mathrm{m}) \mathrm{K}^{+}$through $\sim 100$ quadrupoles, or several beam-plasma oscillations.

The principal array design issues for many parallel channels can be addressed using a reduced number of channels, as long as proper transverse termination of the fields is provided. Thus, a critical next step would be a prototype array with only four or nine channels to gain needed magnet design and fabrication experience.

The final-focus magnets are particularly challenging with the requirement of relatively large aperture (coil winding aperture $\approx 0.2 \mathrm{~m}$ ) and radiation shielding. The strategy fundamentally differs here from the laser IFE approach, where the final optical elements are necessarily in the line of sight of the neutrons, photons, and charged particles emanating from the burning fusion fuel. For the HIF final focusing system, a dipole bend creates a line-ofsight dump for radiation produced by the fusion target. For these final focusing magnets, $\mathrm{Nb}_{3} \mathrm{Sn}$ is the most suitable conductor to meet the focusing requirements, where peak fields in the superconducting material of about $10 \mathrm{~T}$ are desirable [75]. It may be a decade before this developing accelerator technology reaches a mature stage. The required stored energy per unit length is unusually high, so quench protection is very important. The use of shell-type, or racetrack coils is not yet settled, and the design of bundles of converging beam lines (vs subassemblies of parallel focusing quadrupoles with nearby dipoles) awaits resolution. In either case, the incorporation of relatively
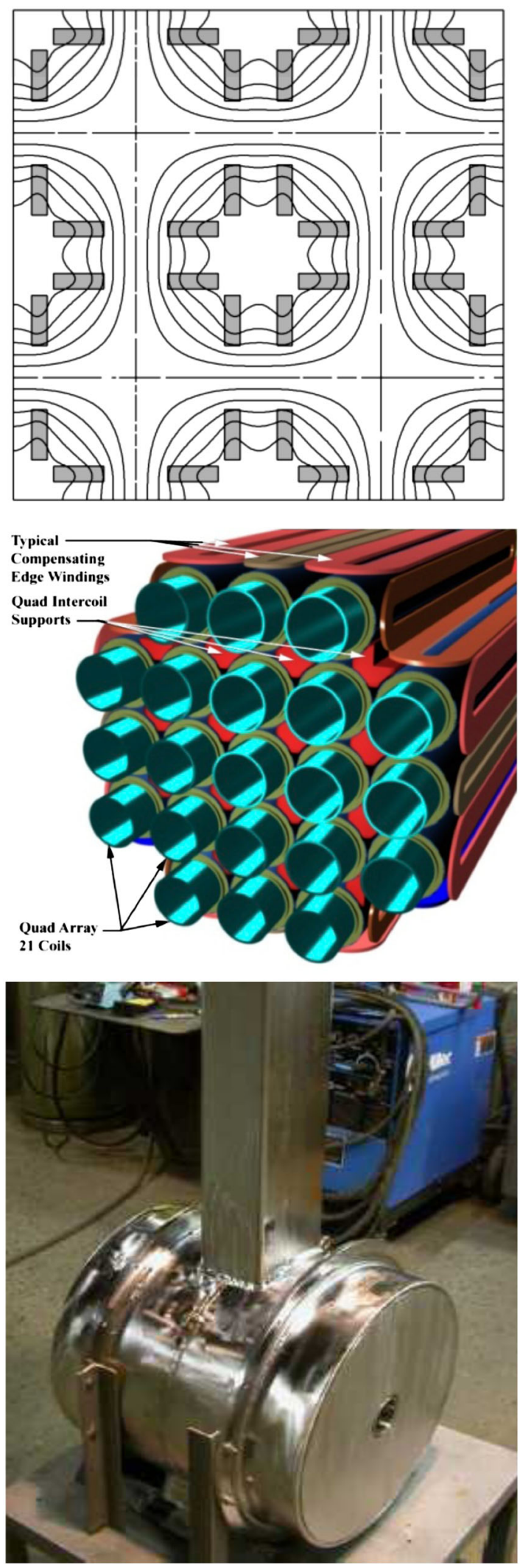

FIG. 5. Transverse coil layout showing magnetic field lines for racetrack coils (top) and the MIT design of a multiplebeam array based on racetrack coils (middle). Photo of the prototype cryostat containing a quadrupole doublet for singlebeam experiments (bottom). 
low-field steering dipoles for small beam centroid corrections must be included.

The working group at the recent Workshop on Accelerators for Heavy Ion Fusion [76] identified the following as important research opportunities for the final-focus magnets: (1) Explore combinations of electrostatic and magnetic quadrupoles for achromatic focusing. (2) Consider elliptical holes in a shield for the final-focus magnets (instead of circular) similar in aspect ratio to the beam shape. (3) Design an array of final-focus magnets with magnetic flux sharing as in the accelerator arrays to achieve a closely packed array of beams. This would allow shielding to be implemented without increasing the size of the array.

To reduce the size of the magnet array, consider different materials for the final magnet, including normal conducting materials based on recently developed materials.

\section{RESEARCH OPPORTUNITIES RELATED TO OTHER FIELDS}

An asset for HIF R\&D is the large rf accelerator community and decades of operating experience of large-scale accelerator facilities worldwide for high-energy and nuclear physics and materials science. Without doubt, an ambitious R\&D program in HIF can only benefit from a greater integration between the rf and much smaller induction accelerator community. A recent Workshop on Accelerators for Heavy Ion Fusion [77] brought together researchers from a number of induction accelerator and $\mathrm{rf}$ accelerator laboratories, including BNL, SNS, LANL, FNAL, and GSI. These and other accelerator laboratories have much expertise in beam dynamics theory and modeling, nonintercepting beam diagnostics, magnet design and fabrication, cryogenics, accelerator control systems, rf and pulsed power. The participants expressed interest in follow-up collaborations.

At LANL, DARHT uses high-current electron induction accelerators [78]. The 2-kA beam current is high enough for beam-loading effects to be tested. Issues of $\mathrm{HV}$ holding and induction-cell design have been tested on DARHT, and it may be possible to design tests or experiments that would address key HIF issues. The expertise accumulated at LANL of designing, operating, and maintaining DARHT is a valuable resource.

Experiments at GSI have shown range shortening of heavy ions in hot matter and more studies are planned to study ion deposition [53] in matter heated quickly by an intense laser.

Experiments with small-scale systems have the potential to mimic the key beam dynamics of an HIF driver. The cost vs benefit of these experiments is attractive because they are able to address important physics questions at relatively low cost. Some experiments might be able to answer key questions related to HIF drivers, and we illustrate this with two examples: Paul traps (e.g., at PPPL and at Hiroshima
University [79-81]), and UMER [21-23,82] are able, in principle, to achieve high space-charge tune depression and transport the beam over the equivalent of thousands of lattice periods.

Paul traps are rf quadrupole traps for charged particles with very low axial velocity. Oscillating quadrupole fields confine the particles transversely, and resemble the strong transverse focusing of particle accelerators. Axially, the charged particles are confined with repulsive barrier fields.

UMER is a high-intensity circular machine $(10 \mathrm{keV}$ electron energy, lattice resembling a storage ring) dedicated to the study of long-path length space-chargedominated beam physics.

Small-scale experiments on these platforms become compelling for HIF development when relevant experiment can be done. To begin with, an intense beam with a space-charge tune depression characteristic of the initial stages of a driver, $\sigma / \sigma_{o}<0.25$ should be injected and matched, and correspond to the space-charge conditions of injected beams described above and in [16]. Thus far, it has been challenging in these small-scale experiments to establish a matched, highly space-charge-dominated beam, where the beam properties are accurately diagnosed.

Another issue is beam quality degradation from resonances that are more likely to be problematic in rings and Paul traps than in linear accelerators. That is because the beams in Paul traps and the UMER ring are subject to identical errors; the beam repeatedly sees the same structures and applied fields, leading to resonances that ruin the beam quality. This resonant behavior might not be seen in a linear induction accelerator, where the beam is continuously being accelerated, and the beam experiences error fields only once per element. However, for Paul traps and UMER, some experiments and modeling so far suggest that it should be possible to design experiments so that the beam properties evolve quickly, and pass through the resonances before damage can be done.

\section{SUMMARY}

There is renewed interest in the development of energy solutions that can provide carbon-free, base-load electricity. The National Ignition Facility campaign of ignition experiments is under way [1]. The U.S. National Academies of Sciences and Engineering (NAS) are reviewing the prospects for inertial confinement fusion energy systems including heavy-ion accelerators [7].

In this paper, the key scientific questions related to HIF drivers and related accomplishments have been summarized. Many of the important scientific and technical questions have been successfully addressed in small experiments computer modeling and theoretical work. Several more questions can be answered in small experiments but what is needed is a big experiment that puts all of this together. The references herein are not exhaustive; an 
extensive bibliography [83] of HIF research through 1996 was compiled, with many more journal publications on HIF since then. Many have appeared in the biennial series of proceedings dedicated to the topic (International Symposium on Heavy Ion Fusion) and additional publications in various journals and conference proceedings.

This paper outlines research to answer key scientific and technical questions in the near term that would be needed for a decision to proceed with a major research facility. The research facility, feasible in 5-10 years, would be required to move forward with a heavy-ion accelerator facility capable of doing HIF target physics and demonstrating at driver scale the essential accelerator components of an HIF power plant, including beam control and focused beam intensity (the IRE). Most of the research is based on a multiple-beam induction linear accelerator, accelerating beams to a final kinetic energy of $1 \mathrm{GeV}$ or higher. Given the combined need for accelerator efficiency and phasespace constraints set by most HIF target designs, and driver cost, it is the best-matched accelerator architecture.

Target physics research must be conducted in parallel because the IRE target physics experimental campaign will drive the accelerator requirements. Some proposed accelerator experiments require modest modifications of existing apparatus, while others would require a greater investment to answer the posed questions.

\section{ACKNOWLEDGMENTS}

The authors would like to thank Grant Logan for his support and encouragement to write this paper. This work was supported by the U.S. Department of Energy under Contracts No. DE-AC02-05CH1123 and No. DE-AC5207NA27344.

[1] See, for example, https://lasers.llnl.gov/.

[2] Induction Accelerators, edited by K. Takayama and R. J. Briggs (Springer-Verlag, Berlin-Heidelberg, 2011).

[3] J. J. Barnard et al., Laser Part. Beams 21, 553 (2003).

[4] J. J. Barnard et al., Nucl. Instrum. Methods Phys. Res., Sect. A 464, 621 (2001) [http://escholarship.org/uc/item/ $7 \mathrm{r} 43 \mathrm{j} 8 \mathrm{sp}]$.

[5] J. Hovingh, V. O. Brady, A. Faltens, D. Keefe, and E. P. Lee, Fusion Technol. 13, 255 (1988).

[6] R. W. Moir, Part. Accel. 37-38, 467 (1992) [http://hifweb .lbl.gov/public/Sharp/HIF_documents/Moir-HYYLIFE \%20II\%20design.pdf]; R. Abbott, S. Pemberton, P. F. Peterson, G.-P. Sun, P. Wright, R. Holmes J. Latkowski, R. Moir, and K. Springer, Fusion Technol. 39, 732 (2001); Raluca O. Scarlat and Per F. Peterson, "The current status of fluoride salt cooled high temperature reactor (FHR) technology and its overlap with HIF target chamber concepts," Nucl. Instrum. Methods Phys. Res., Sect. A (to be published).

[7] An Assessment of the Prospects for Inertial Fusion Energy (2012) [http://www.nap.edu].
[8] Prospects for Inertial Confinement Fusion Energy Systems, The National Academies Board on Physics and Astronomy, Washington, DC [http://sites.nationalacademies .org/BPA/BPA_058425].

[9] R. Bangerter, G. Navratil, and N. Sauthoff, J. Fusion Energy 20, 75 (2001) [http://link.springer.com/article/ 10.1023/A\%3A1021361624409?null].

[10] B. G. Logan, Ion-Beam-Driven Inertial Fusion Energy, at the Second Meeting of the National Academies of Sciences and Engineering, 2011 [http://fire.pppl.gov/ IFE_NAS2_HIF_Logan.pdf].

[11] L. R. Prost et al., Phys. Rev. ST Accel. Beams 8, 020101 (2005).

[12] T. J. Fessenden, Nucl. Instrum. Methods Phys. Res., Sect. A 278, 13 (1989); M. G. Tiefenback and D. Keefe, IEEE Trans. Nucl. Sci. 32, 2483 (1985).

[13] C. M. Celata, A. Faltens, D. L. Judd, L. Smith, and M. G. Tiefenback, in Proceedings of the 1987 Particle Accelerator Conference (IEEE, Washington, DC, 1987) [http://accelconf .web.cern.ch/accelconf/p87/PDF/PAC1987_1167.PDF].

[14] I. Haber, D. Kehne, M. Reiser, and H. Rudd, Phys. Rev. A 44, 5194 (1991).

[15] P. A. Seidl, C. Celata, A. Faltens, E. Henestroza, and S. MacLaren, Phys. Rev. ST Accel. Beams 6, 090101 (2003).

[16] J. W. Kwan, IEEE Trans. Plasma Sci. 33, 1901 (2005).

[17] S. A. MacLaren, A. Faltens, P. A. Seidl, and D. V. Rose, Phys. Plasmas 9, 1712 (2002).

[18] P. K. Roy, S. S. Yu, S. Eylon, et al., Phys. Plasmas 11, 2890 (2004).

[19] A. B. Sefkow, R.C. Davidson, E.P. Gilson, I. D. Kaganovich, A. Anders, J. E. Coleman, M. Leitner, S. M. Lidia, P. K. Roy, P. A. Seidl, W. L. Waldron, S. S. Yu, and D. R. Welch, Phys. Plasmas 16, 056701 (2009).

[20] P. K. Roy, S.S. Yu, E. Henestroza, A. Anders, F. M. Bieniosek, J. Coleman, S. Eylon, W. G. Greenway, M. Leitner, B. G. Logan, W. L. Waldron, D. R. Welch, C. Thoma, A. B. Sefkow, E.P. Gilson, P.C. Efthimion, and R.C. Davidson, Phys. Rev. Lett. 95, 234801 (2005).

[21] B. Beaudoin, I. Haber, R. A. Kishek, S. Bernal, T. Koeth, D. Sutter, P. G. O'Shea, and M. Reiser, Phys. Plasmas 18, 013104 (2011).

[22] T.W. Koeth et al., in Proceedings of the 2011 Particle Accelerator Conference, NY, USA (IEEE, New York, 2011) [http://accelconf.web.cern.ch/AccelConf/PAC2011/ papers/moobs3.pdf].

[23] B. Beaudoin, S. Bernal, K. Fiuza, I. Haber, R. A. Kishek, T. Koeth, M. Reiser, D. Sutter, and P. G. O'Shea, in Proceedings of the 2011 Particle Accelerator Conference, NY, USA (Ref. [23]) [http://accelconf.web .cern.ch/AccelConf/PAC2011/papers/moods1.pdf].

[24] L. Ahle et al., in Proceedings of the 18th Particle Accelerator Conference, New York, 1999 (IEEE, New York, 1999) [http://accelconf.web.cern.ch/AccelConf/p99/ PAPERS/THP124.PDF].

[25] T. C. Sangster et al., Nucl. Instrum. Methods Phys. Res., Sect. A 415, 310 (1998).

[26] W. M. Fawley, T. Garvey, S. Eylon, E. Henestroza, A. Faltens, T. J. Fessenden, K. Hahn, L. Smith, and D. P. Grote, Phys. Plasmas 4, 880 (1997). 
[27] A. Friedman, Nucl. Instrum. Methods Phys. Res., Sect. A 544, 160 (2005).

[28] D. P. Grote, A. Friedman, J.-L. Vay, and I. Haber, AIP Conf. Proc. 749, 55 (2005).

[29] J.-L. Vay, D. P. Grote, R. H. Cohen, and A. Friedman, Comput. Sci. Discovery 5, 014019 (2012).

[30] D. R. Welch, D. V. Rose, B. V. Oliver, and R. E. Clark, Nucl. Instrum. Methods Phys. Res., Sect. A 464, 134 (2001).

[31] H. Qin, E. A. Startsev, and R. C. Davidson, Phys. Rev. ST Accel. Beams 6, 014401 (2003).

[32] M. Kireeff-Covo, A. Molvik, A. Friedman, J.-L. Vay, P. Seidl, G. Logan, D. Baca, and J. Vujic, Phys. Rev. Lett. 97, 054801 (2006); R. Cohen, A. Friedman, M. Kireeff Covo, S. M. Lund, A. W. Molvik, F. M. Bieniosek, P. A. Seidl, J.-L. Vay, P. Stoltz, and S. Veitzer, Phys. Plasmas 12, 056708 (2005).

[33] J. F. Latkowski and W. R. Meier, Fusion Sci. Technol. 44, 2, 300 (2003) [http://www.new.ans.org/pubs/journals/fst/ a_351].

[34] See, for example, Brookhaven National Laboratory Report No. BNL-50769. Proceedings and workshops from most Heavy Ion Fusion symposia and workshops are available at http://ahif.lbl.gov/relevant-papers.

[35] J. Sovey et al., Report No. NASA/TM-1999-209439, 1999 [http://www.scribd.com/doc/46736439/A-Synopsis-of-IonPropulsion-Development-Projects-in-the-US-SERT-1to-Deep-Space-I].

[36] F. M. Bieniosek, C. Celata, E. Henestroza, J. Kwan, L. Prost, P. Seidl, A. Friedman, D. Grote, S. Lund, and I. Haber, Phys. Rev. ST Accel. Beams 8, 010101 (2005).

[37] G. A. Westenskow, D. Grote, F. M. Bieniosek, and J. W. Kwan, in Proceedings of the 2007 Particle Accelerator Conference, Albuquerque, New Mexico (IEEE, New York, 2007) [http://accelconf.web.cern.ch/ AccelConf/p07/PAPERS/FRYAB01.PDF].

[38] A. Faltens and D. Keefe, in Proceedings of the Heavy Ion Fusion Workshop, Berkeley, 1979, pp. 157-181 (Reports No. SLAC-R-542, No. LBL-10301, and No. CONF7910122) [http://slac.stanford.edu/pubs/slacreports/reports01/ slac-r-542.pdf].

[39] Selected references for HIF related sources and injectors are available at http://ahif.lbl.gov/selected-references/ sources-and-injectors.

[40] W. Chupp et al., in Proceedings of the Particle Accelerator Conference (IEEE, New York, 1979), http:// accelconf.web.cern.ch/AccelConf/p79/PDF/PAC1979_3036 .PDF.

[41] Igor D Kaganovich, E. Startsev, and R. C. Davidson, New J. Phys. 8, 278 (2006).

[42] S. M. Lund and B. Bukh, Phys. Rev. ST Accel. Beams 7, 024801 (2004).

[43] R. H. Cohen, A. Friedman, S. Lund, A. Molvik, E. Lee, T. Azevedo, J.-L. Vay, P. Stoltz, and S. Veitzer, Phys. Rev. ST Accel. Beams 7, 124201 (2004).

[44] Steven M. Lund, David P. Grote, and Ronald C. Davidson, Nucl. Instrum. Methods Phys. Res., Sect. A 544, 472 (2005).

[45] E. A. Startsev, .C. Davidson, and H. Qin, Phys. Rev. ST Accel. Beams 8, 124201 (2005).

[46] C. M. Celata et al., in Proceedings of the Inertial Fusion Sciences and Applications 2003 (IFSA 2003), Monterey,
CA [http://www.escholarship.org/uc/item/2b31h7dm]; M. Leitner et al., in Proceedings of the 20th Particle Accelerator Conference, Portland, OR, 2003 (IEEE, New York, 2003) [http://accelconf.web.cern.ch/AccelConf/ p03/PAPERS/TPAG040.PDF].

[47] 2002 Fusion Summer Study, Snowmass, USA, 2002 [https://fusion.gat.com/conferences/snowmass/].

[48] I. Hofmann and I. Bozsik, in Proceedings of the Symposium on Accelerator Aspects of Heavy Ion Fusion (1982), p. 362, GSI-82-8.

[49] I. Haber, in Proceedings of the Symposium on Accelerator Aspects of Heavy Ion Fusion (1982), p. 372, GSI-82-8.

[50] M. Venturini, Phys. Rev. Lett. 81, 96 (1998).

[51] J. J. Barnard, J. Miller, and I. Haber, in Proceedings of the Particle Accelerator Conference, Washington, DC, 1993 (IEEE, New York, 1993) [http://accelconf.web.cern.ch/ accelconf/p93/PDF/PAC1993_3612.PDF].

[52] R. O. Bangerter, "Heavy Ion Fusion Targets; Issues for Fast Ignition," Nucl. Instrum. Methods Phys. Res., Sect. A (to be published); John Barnard, John Perkins, Dave Bailey, Roger Bangerter, Alex Burke, Darwin Ho, Dieter Hoffmann, Shigeo Kawata, Grant Logan, and Matt Terry, in the Workshop on Accelerators for Heavy Ion Inertial Fusion, Lawrence Berkeley National Laboratory, 2011 [http://ahif.lbl.gov].

[53] A. Frank et al., Phys. Rev. E 81, 026401 (2010), and references therein.

[54] D. Callahan-Miller and M. Tabak, Phys. Plasmas 7, 2083 (2000).

[55] S. S. Yu, W. R. Meier, R. P. Abbott, J. J. Barnard, T. Brown, D. A. Callahan, C. Debonnel, P. Heitzenroeder, J.F. Latkowski, B. G. Logan, S. J. Pemberton, P. F. Peterson, D. V. Rose, G.-L. Sabbi, W. M. Sharp, and D. R. Welch, Fusion Sci. Technol. 44, 266 (2003) [http://escholarship .org/uc/item/6vq5x9x8].

[56] D. A. Callahan-Miller and M. Tabak, Nucl. Fusion 39, 1547 (1999).

[57] D. Callahan, M. Herrmann, and M. Tabak, Laser Part. Beams 20, 405 (2002) [http://dx.doi.org/10.1017/ S0263034602203079].

[58] D. T. Goodin et al., Nucl. Fusion 44, S254 (2004).

[59] D. J. Meeker and R. O. Bangerter, Lawrence Livermore National Laboratory Report No. UCRL-50021-81, 1982.

[60] G. O. Allshouse, R. E. Olson, D. A. Callahan-Miller, and M. Tabak, Nucl. Fusion 39, 893 (1999).

[61] T. Someya, K. Miyazawa, T. Kikuchi, and S. Kawata, Laser Part. Beams 24, 359 (2006) [http://dx.doi.org/ 10.1017/S0263034606060526].

[62] B. Logan, L. Perkins, and J. Barnard, Phys. Plasmas 15, 072701 (2008).

[63] N. A. Tahir and K. A. Long, Phys. Fluids 29, 1282 (1986).

[64] M. Basko and J. Meyer-ter-Vehn, Nucl. Fusion 33, 601 (1993).

[65] M. Terry, J. J. Barnard, and J. Perkins (to be published).

[66] M. M. Basko, M. D. Churasov, and A. G. Aksenov, Laser Part. Beams 20, 411 (2002) [http://dx.doi.org/10.1017/ S0263034602203080].

[67] E. Henestroza and B. G. Logan, Phys. Plasmas 19, 072706 (2012).

[68] A. W. Chao and M. Tigner, Accelerator Physics and Engineering (World Scientific, Singapore, 1999), Sec. 1.6.5. 
[69] C. Peters et al., in Proceedings of LINAC 2000 [http:// arxiv.org/abs/hep-ex/0009062v1].

[70] George J. Caporaso et al., in Proceedings of the 23rd Particle Accelerator Conference, Vancouver, Canada, 2009 (IEEE, Piscataway, NJ, 2009) [http://accelconf.web .cern.ch/AccelConf/PAC2009/papers/th3gai02.pdf].

[71] L. L. Reginato, IEEE Trans. Nucl. Sci. 30, 2970 (1983).

[72] S. Eylon, E. Henestroza, S. M. Lidia, D. L. Vanecek, S. S. Yu, T. L. Houck, G. A. Westenskow, and D. E. Anderson, in Proceedings of the 18th Particle Accelerator Conference, New York, 1999 (Ref. [24]) [http://accelconf .web.cern.ch/accelconf/p99/PAPERS/FRA3.PDF].

[73] R. O. Bangerter et al., IEEE Trans. Appl. Supercond. 13, 1530 (2003).

[74] Chen-yu Gung, N. N. Martovetsky, R. R. Manahan, J. V. Minervini, J. H. Schultz, G. Sabbi, and P. A. Seidl, IEEE Trans. Appl. Supercond. 15, 1171 (2005).

[75] T. Brown, G.-L. Sabbi, J. J. Barnard, P. Heitzenroeder, J. Chun, J. Schmidt et al., at the 15 th Topical Meeting on the Technology of Fusion Energy, 2002 Washington, D.C. [http://escholarship.org/uc/item/73t3b31t].

[76] See the Chamber and Chamber Driver Interface working group summary, Workshop on Accelerators for Heavy
Ion Fusion, 2011, Berkeley, USA [http://ahif.lbl.gov/ workshop-reports].

[77] Workshop on Accelerators for Heavy Ion Fusion, 2011, Lawrence Berkeley National Laboratory [http://ahif.lbl .gov/executive-summary].

[78] S. Nath, in Proceedings of the LINAC 2010 [http://accelconf .web.cern.ch/AccelConf/LINAC2010/papers/th304.pdf].

[79] M. Chung, E. P. Gilson, R. C. Davidson, P. C. Efthimion, and R. Majeski, Phys. Rev. Lett. 102145003 (2009).

[80] E. P. Gilson, R. C. Davidson, P.C. Efthimion, and R. Majeski, Phys. Rev. Lett. 92, 155002 (2004).

[81] S. Ohtsubo, M. Fujioka, H. Higaki, K. Ito, H. Okamoto, H. Sugimoto, and S. M. Lund, Phys. Rev. ST Accel. Beams 13, 044201 (2010).

[82] I. Haber, S. Bernal, B. Beaudoin, M. Cornacchia, D. Feldman, R. B. Feldman, R. Fiorito, K. Fiuza, T. F. Godlove, R. A. Kishek, P. G. O'Shea, B. Quinn, C. Papadopoulos, M. Reiser, D. Stratakis, D. Sutter, J. C. T. Thangaraj, K. Tian, M. Walter, and C. Wu, Nucl. Instrum. Methods Phys. Res., Sect. A 606, 64 (2009).

[83] T. F. Godlove, Fusion Eng. Des. 32-33, 25 (1996). The bibliography can be found at http://ahif.lbl.gov/selectedreferences. 